\title{
Editorial
}

\section{Advances in Time Series Analysis and Its Applications}

\author{
Zhong-Ke Gao, ${ }^{1}$ Michael Small, ${ }^{2,3}$ Reik Donner, ${ }^{4}$ Du Meng, ${ }^{5}$ and Hamed O. Ghaffari ${ }^{6}$ \\ ${ }^{1}$ School of Electrical Engineering and Automation, Tianjin University, Tianjin 300072, China \\ ${ }^{2}$ School of Mathematics and Statistics, University of Western Australia, Crawley, WA 6009, Australia \\ ${ }^{3}$ Mineral Resources, CSIRO, Kensington, WA 6151, Australia \\ ${ }^{4}$ Potsdam Institute for Climate Impact Research, 14473 Potsdam, Germany \\ ${ }^{5}$ Tianjin University of Science and Technology, Tianjin 300222, China \\ ${ }^{6}$ Department of Civil Engineering, University of Toronto, Toronto, ON, Canada M5S 3E3 \\ Correspondence should be addressed to Zhong-Ke Gao; zhongkegao@tju.edu.cn
}

Received 3 July 2016; Accepted 3 July 2016

Copyright (C) 2016 Zhong-Ke Gao et al. This is an open access article distributed under the Creative Commons Attribution License, which permits unrestricted use, distribution, and reproduction in any medium, provided the original work is properly cited.

This special issue is particularly focused on new theories and applications of time series analysis. Characterizing dynamical processes in a time-dependent complex system from observed time series of just one or at most a few variables is a fundamental problem of significant importance in many research fields. Time series analysis has been broadly adopted in scientific research and engineering applications. Many theoretical developments and new methods for time series analysis have significantly contributed to the understanding of complex systems. However, when system complexity increases, it becomes difficult to describe the dynamic behavior from time series and traditional time series analysis methods have difficulty coping with the specific burdens of this increased complexity. There are still many challenges in the development of time series analysis methods addressing such problems. Advanced methods of time series analysis provide new prospects for investigating complex systems and will result in substantial and sustainable deepening of our knowledge in a broad variety of fields of science. This special issue covers the several emerging and important research areas: (1) "Application of AR Model in the Analysis of Preearthquake Ionospheric Anomalies"; (2) "Chebyshev Similarity Match between Uncertain Time Series"; (3) "Comparison of Stabilization Ability of Models for Hydrological Time Series with a Deterministic Trend"; (4) "Cost-Sensitive Estimation of ARMA Models for Financial Asset Return Data"; (5) "Short-Term Traffic Flow Local Prediction Based on Combined Kernel Function Relevance Vector Machine Model"; (6) "Incremental Discriminant Analysis on Interval-Valued Parameters for Emitter Identification"; (7) "An Improved Nonlinear Grey Bernoulli Model Combined with Fourier
Series"; (8) "Spatial Information Entropy and Its Application in the Degradation State Identification of Hydraulic Pump"; (9) "Human Activity Recognition as Time-Series Analysis"; (10) "The Multiscale Conformation Evolution of the Financial Time Series"; (11) "Low-Frequency Volatility in China's Gold Futures Market and Its Macroeconomic Determinants"; (12) "Application of Passivity-Based Control and Time-Frequency Representation in a Doubly Fed Induction Generator System"; (13) "Applying Data Mining Techniques to Identify Suitable Activities"; (14) "Parallel kd-Tree Based Approach for Computing the Prediction horizon Using Wolf's Method"; (15) "Relationship between Chinese and International Crude Oil Prices: A VEC-TARCH Approach"; (16) "Transportation Mode Detection Based on Permutation Entropy and Extreme Learning Machine"; and (17) "Intuitionistic Fuzzy Time Series Forecasting Model Based on Intuitionistic Fuzzy Reasoning." The range of both methods and applications demonstrates the vitality of this research area. We hope that our special issue offers a timely view of the advances in time series analysis and its applications.

\section{Acknowledgments}

We sincerely express our gratitude to the authors for their contributions and the reviewers for their insightful comments.

Zhong-Ke Gao Michael Small

Reik Donner

Du Meng

Hamed O. Ghaffari 


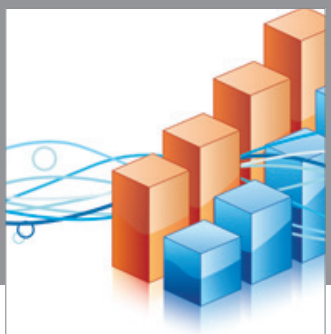

Advances in

Operations Research

vatem alat4

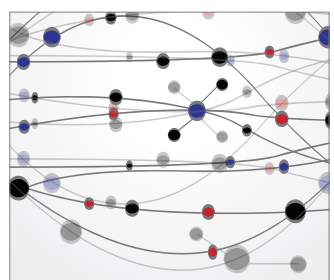

\section{The Scientific} World Journal
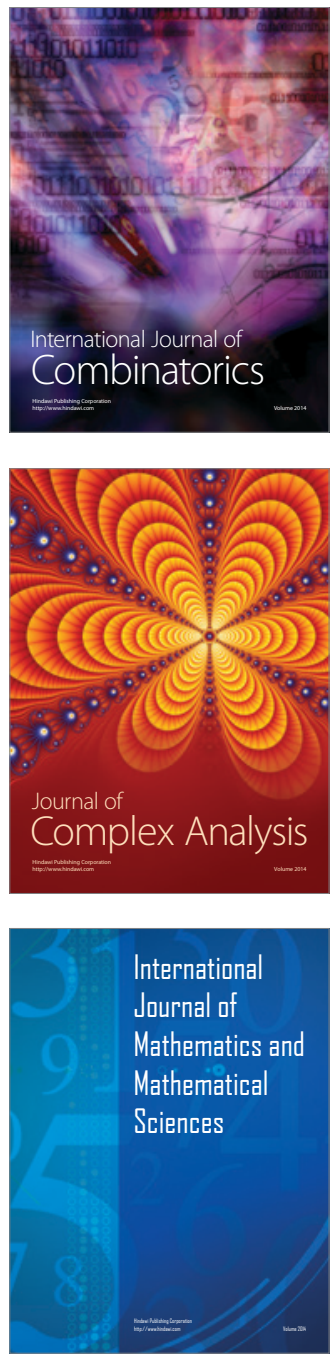
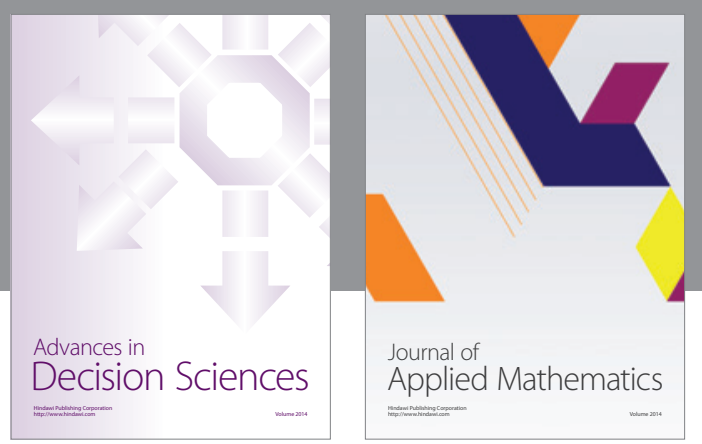

Algebra

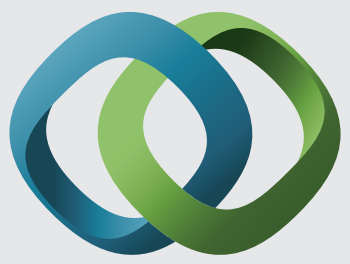

\section{Hindawi}

Submit your manuscripts at

http://www.hindawi.com
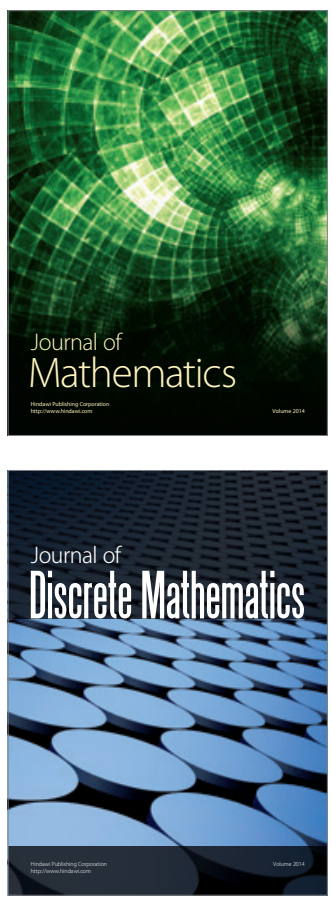

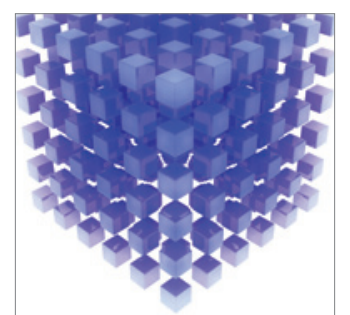

Mathematical Problems in Engineering
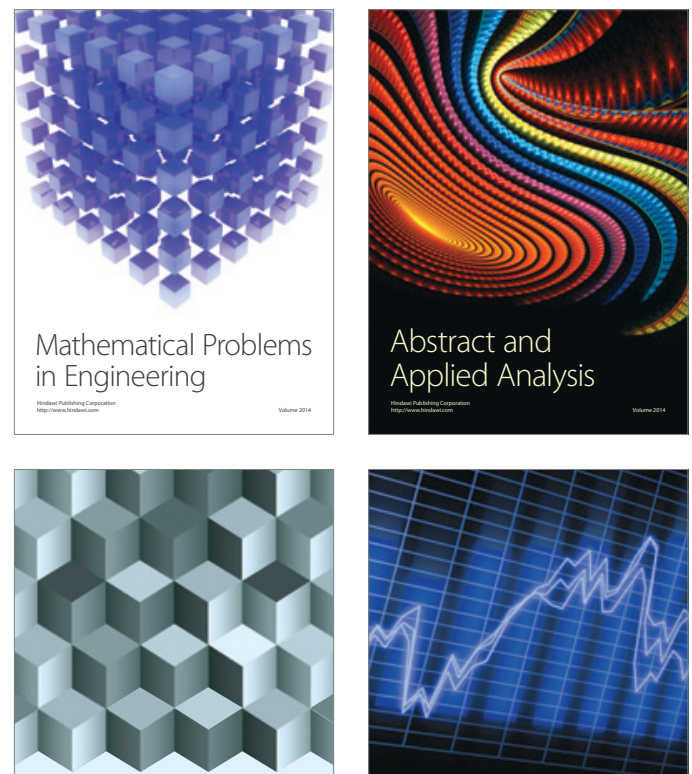

Journal of

Function Spaces

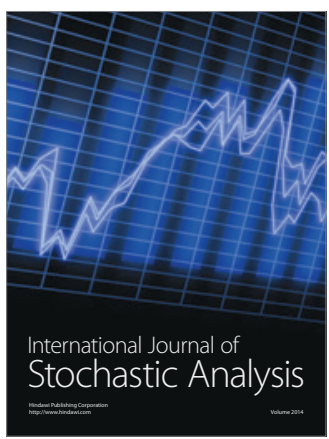

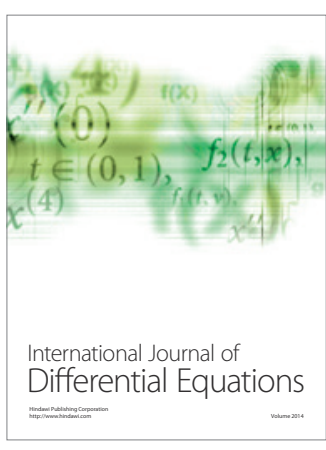
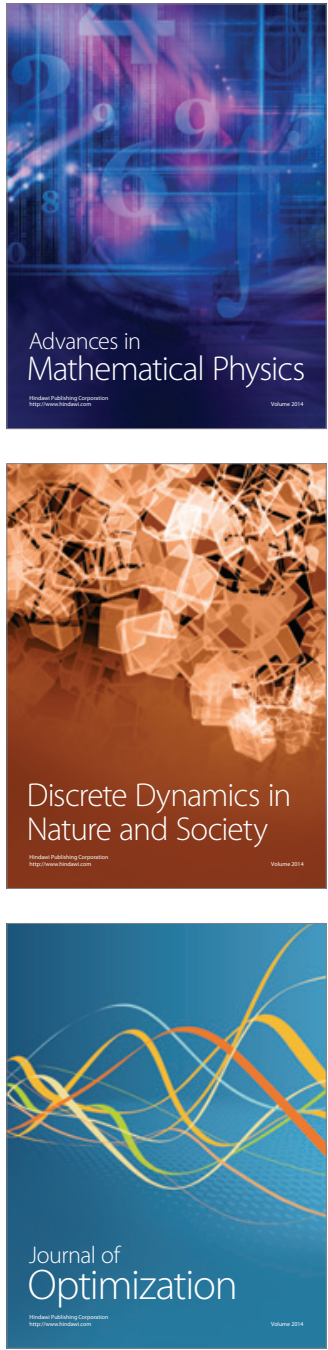